\title{
EXISTENCE OF POSITIVE SOLUTIONS FOR A SINGULAR PROBLEMS OF CAFFARELLI-KOHN-NIRENBERG-LIN TYPE
}

\section{NEMAT NYAMORADI}

Abstract. In this paper, we study the existence of nontrivial critical points of the functional

$$
\begin{array}{r}
J_{\lambda, \mu}(u, v)=\frac{1}{p} \int_{\mathbb{R}^{N}}\left(\left.\left.|| x\right|^{-a} \nabla^{k} u\right|^{p}-\left.\left.\lambda f(x)|| x\right|^{-(a+k)} u\right|^{p}\right) d x+\frac{1}{q} \int_{\mathbb{R}^{N}}\left(\left.\left.|| x\right|^{-a} \nabla^{k} v\right|^{q}\right. \\
\left.-\left.\left.\mu g(x)|| x\right|^{-(a+k)} v\right|^{q}\right) d x-\int_{\mathbb{R}^{N}} h(x)|x|^{-b(\alpha+\beta+2)}|u|^{\alpha+1}|v|^{\beta+1} d x,
\end{array}
$$

related to the Caffarelli-Kohn-Nirenberg inequality and its higher order variant by Lin.

Mathematics subject classification (2010): 35A15, 35B33, 35J70. function.

Keywords and phrases: Nehari manifold, Caffarelli-Kohn-Nirenberg inequality, sign-changing weight

\section{REFERENCES}

[1] C. O. Alves, A. El Hamidi, Nehari manifold and existence of positive solutions to a class of quasilinear problems, Nonlinear Anal., 60 (2005), 611-624.

[2] R. B. AssunÇão, P. C. CARRIÃo, O. H. MiYAGaKI, Subcritical perturbations of a singular quasilinear elliptic equation involving the critical Hardy-Sobolev exponent, Nonlinear Anal., 66 (2007), 1351-1364.

[3] P. A. Binding, P. DrabeK, Y. X. HuAng, On Neumann boundary value problems for some quasilinear elliptic equations, Electron. J. Differential Equations, 5 (1997), 1-11.

[4] K. J. BROWN, The Nehari manifold for a semilinear elliptic equation involving a sublinear term, Calc. Var., 22 (2005), 483-494.

[5] K. J. BROWN, Y. ZHANG, The Nehari manifold for a semilinear elliptic problem with a sign changing weight function, J. Differential Equations, 193 (2003), 481-499.

[6] K. J. BRown, T. F. WU, A fibering map approach to a semilinear elliptic boundary value problem, Electron. J. Differential Equations, 69 (2007), 1-9.

[7] K. J. BRown, T. F. WU, Multiplicity of positive solution of p-Laplacian problems with sign-changing weight function, Int. J. Math. Anal., 1, 12 (2007), 557-563.

[8] K. J. BRown, T. F. WU, A semilinear elliptic system involving nonlinear boundary condition and sign changing weight function, J. Math. Anal. Appl., 337 (2008), 1326-1336.

[9] L. CAFFARELLI, R. KoHn, L. NirenBerG, First order interpolation inequalities with weights, Compos. Math., 53 (1984), 259-275.

[10] T. S. Hsu, Multiple positive solutions for a critical quasilinear elliptic system with concave-convex nonlinearities, Nonlinear Anal., 71 (2009), 2688-2698.

[11] L. HuAng, X. P. Wu, C. L. TANG, Existence and multiplicity of solutions for semilinear elliptic equations with critical weighted Hardy-Sobolev exponents, Nonlinear Anal., 71 (2009), 1916-1924.

[12] D. KANG, Nontrivial solutions to semilinear elliptic problems involving two critical Hardy-Sobolev exponents, Nonlinear Anal., 72 (2010), 4230-4243.

[13] C. S. Lin, Interpolation inequalities with weights, Comm. PDE, 11 (1986), 1515-1538.

[14] Z. NehARI, On a class of nonlinear second-order differential equations, Trans. Amer. Math. Soc., 95 (1960), 101-123. 
[15] S. Waliullah, Higher order singular problems of Caffarelli-Kohn-Nirenberg-Lin type, J. Math. Anal. Appl., 385 (2012), 721-736.

[16] M. Willem, Minimax Theorems, Boston, Basel, Berlin, 1996.

[17] T. F. WU, On semilinear elliptic equations involving concave-convex nonlinearities and sign-changing weight function, J. Math. Anal. Appl., 318 (2006), 253-270.

[18] B. XUAN, The solvability of quasilinear Brezis-Nirenberg-type problems with singular weights, Nonlinear Anal., 62 (2005), 703-725. 